Research Article

\title{
Minimizing Zapping Delay Using Adaptive Channel Switching with Personalized Electronic Program Guide
}

\author{
Timothy T. Adeliyi $\mathbb{D}^{D}$, Oludayo O. Olugbara $\mathbb{D}$, and Steven Parbanath \\ ICT and Society Research Group, Luban Workshop, Durban University of Technology, Durban, South Africa \\ Correspondence should be addressed to Timothy T. Adeliyi; tim.adeliyi@gmail.com
}

Received 1 November 2020; Revised 6 July 2021; Accepted 4 September 2021; Published 21 September 2021

Academic Editor: Patrizia Grifoni

Copyright (c) 2021 Timothy T. Adeliyi et al. This is an open access article distributed under the Creative Commons Attribution License, which permits unrestricted use, distribution, and reproduction in any medium, provided the original work is properly cited.

\begin{abstract}
The pervasive acceptability of a revolution from monodirectional push-based media broadcasting to a bidirectional interactive pull-based internet protocol television (IPTV) has spotted significant development in recent years. The pervasive acceptability is because of the mammoth number of exhilarating television (TV) channels that IPTV offers. However, the channel switching feature of a TV system requires additional development despite the increased implementation of IPTV systems worldwide. Subscribers of IPTV services must be able to swiftly explore live TV stations and video contents of interest seamlessly, but zapping delay is a deterrent that occurs during a channel change that causes a significant glitch in IPTV systems. Many of the literature approaches such as channel prediction based on behavior analysis have shown flaws in resolving zapping delay. The approach of this study uses adaptive channel switching with a personalized electronic program guide to resolving zapping delay. The resolution saves the subscribers the time of channel navigation by eliminating the need to search for channels they want to view.
\end{abstract}

\section{Introduction}

The pervasiveness of internet protocol television (IPTV) coupled with the evolution of digital broadcasting services is rapidly emerging as a substitute for orthodox television (TV) broadcasting delivery systems [1]. IPTV provides the users with a compelling bundle for a wide variety of stirring applications, allowing significant suppleness to network, content providers, and serving as a lucrative source of revenue. IPTV services can be appositely separated into two primary types, which are live video streaming and video-ondemand $(\mathrm{VoD})$. Due to bandwidth constraints, live TV is broadcasted across a multicast network after the raw video has been encoded and relayed to subscribers ondemand. The VoD is a stored media material that is distributed to subscribers on request [2]. However, subscribers continue to have difficulty in selecting the desired channels to view because of zapping delay. This is despite the intrinsic benefits of IPTV providing many choices of live TV channels across different categories of digital multimedia in various nations. IPTV network providers must ensure a greater degree of experience than the orthodox broadcast TV services. Subscribers of IPTV multichannel systems tend to switch between TV channels in fewer minutes because of the variety of channel contents being aired, innate curiosity, or preference to bypass commercial breaks [3]. They tend to change between TV channels by pressing channel numbers on the remote control, sequentially pressing remote control buttons, or utilizing up and down remote buttons [4]. The other important ways to change channels are using an electronic program guide and navigating amid channels in the same, which are the least popular options with each receiving about $4 \%$ of popularity votes.

Different ways have been proposed in the literature to assist subscribers in swiftly locating their preferred channels of interest. They include a recommendation system, an electronic program guide (EPG), and a search engine $[5,6]$. The "advanced natural language processing, machine learning techniques, collaborative filtering, data mining, information retrieval, and multimedia content analytics are all used in a recommendation system" [7]. These approaches were created to automatically generate recommendations for 
subscribers by recommending channels of interest. Amazon, MovieLens, Netflix, and Spotify are among the organizations that have implemented different recommendation systems to improve the quality of experiences for their subscribers [8]. Unger [9] has claimed that recommendation systems may generate privacy issues because network providers are aware of subscriber content that can be extracted. After all, recommendation systems rely only on information such as history logs collected obstructive or unobstructively from subscribers.

Search engines are used to match content searched for by subscribers to identify TV channels and other IPTV contents. However, they cannot recommend a preferred channel or content that subscribers prefer to watch in most cases. In addition, EPG which is a multilayer program schedule is used to assist subscribers in channel switching. It contains program information such as titles, names, exact start and end times, and playing periods. It serves as a guide in an IPTV multichannel environment saved on the IPTV settop box. The ability to receive schedule information for both current and future events of TV networks is boosting the value of this service [10-12]. Due to the aforementioned challenges faced by channel recommendation systems in IPTV, an approach based on adaptive channel switching with an electronic program guide is reported in this paper that can help to minimize zapping delay in IPTV at the subscriber level. Hence, reducing the total seek and distance time of the channel to the barest minimum. The remainder of this paper is succinctly summarized as follows. Related works are reviewed in Section 2, which is followed by the proposed approach in Section 3. The implementation of the proposed approach is explicated in Section 4, and the paper is briefly concluded in Section 5 .

\section{Related Works}

Zapping delay is a significant deterrent in IPTV for which various strategies and approaches have been proposed. Context-aware recommender systems deal with modeling subscriber perceptions, preferences, and integrating the existing contextual information such as time and place in contrast to orthodox recommendation algorithm [13]. Song et al. [14] presented a context-aware recommendation system for decreasing zapping delay in the next-generation network architecture to provide IPTV services. The suggested recommendation system incorporates explicit and implicit preferences of subscribers by introducing weight values across nodes along the various levels to correlate the choice of subscribers with context information. The findings of their strategy were promising in terms of personalized content selection, service initiation delays, and EPG browsing time. However, the quality of experience received by subscribers was not evaluated. A context-aware personalized program guide based on a neural network of viewing habits of subscribers has been proposed to predict whether a subscriber is excited about a given program being viewed on a channel [15]. TV channel prefetching approach based on channel popularity was introduced to reduce zapping latency in IPTV and improve the quality of experience for subscribers [16, 17]. Bahn [18] offered hybrid techniques that agglutinate channel prefetching and reordering methods to reduce zapping delay in IPTV.

The adaptive buffering method that exploits two adaptive buffers was proposed for the zapping time reduction [19]. The impetus behind a wholly intelligent personalized EPG is that it is intended to assess behavior of subscribers in recommending interested channel programs [20, 21]. The deployment of one fast channel change (FCC) server in the internet protocol (IP) backbone to send a unicast stream to the set-top box (STB) before sending the standard multicast stream after each channel change was proposed as a channel change acceleration method delivery [22]. However, due to massive unicast data delivered by FCC servers to STBs, installing such a method would result in excessive network bandwidth utilization [23]. Consequently, Khabbiza [24] offered a novel technique to lessen zapping latency by utilizing a peer-to-peer strategy that reduces unicast traffic bandwidth utilization. The researchers advocated deploying FCC servers at the subscriber level instead of implementing a rapid channel change server on the IP backbone. This implies that one STB will receive unicast traffic from another STB rather than from the central server. This strategy will generate buffer overrun on the STB of a subscriber, which will result in zapping delay.

It is understandably difficult for a network provider to deliver a reduced channel zapping latency as a feature to consumers of an IPTV system, but it is unavoidable a requirement for customer quality of experience (QoE) [25]. Hence, many media studies have recently adopted EPG personalization by utilizing context-aware recommendation algorithms to generate TV shows for subscribers to suit their needs and improve media experience. Sailaja [26] investigated the attitudes of subscribers toward a customized electronic program guide that employs a recommendation algorithm that accepts the personal information of a subscriber as input. However, according to the study findings, subscribers highly rated the accruing capabilities afforded by digital broadcast personalization, but they were concerned about the consequences of their recovered private information, particularly with regard to their geographical position. This assertion is intrinsically an inspiration for this paper to give subscribers the freedom of choice to customize their EPG with a reminder without intruding into their private details. This is an approach that IPTV subscribers have anticipated to enjoy the inherent benefits of a personalized EPG without having their private information misused to improve their media experience.

\section{Proposed Approach}

The novel adaptive channel switching with a personalized electronic program guide is being proposed in this paper based on four modular methods as shown in Figure 1. These modular methods are a two-list scheme, personalized electronic program guide, explicit semantic analysis of bag-ofwords, and rich simple syndication (RSS). 


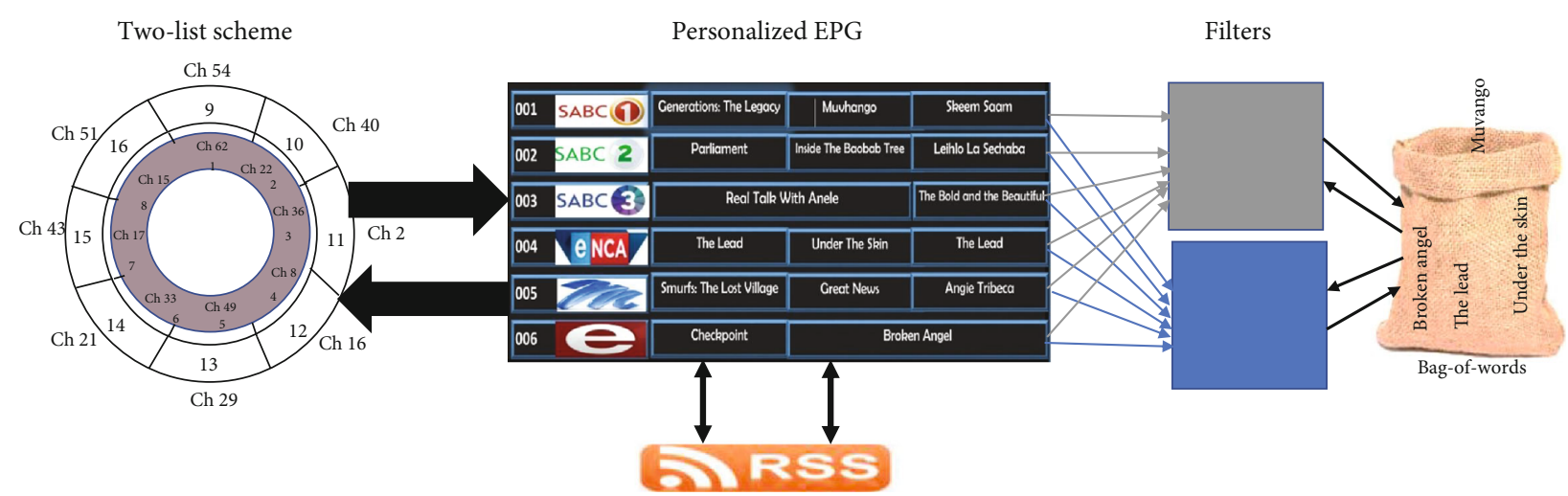

Figure 1: Adaptive channel switching.

3.1. Two-List Scheme. The two-list scheme at the subscriber level is the first modular method of the adaptive channel switching framework $[27,28]$. The method encourages faster channel switching and reduces zapping delay by minimizing the total search distance and total seek time. Selecting the desired TV show from several hundreds of channels accessible on IPTV can be intractably difficult, resulting in a higher channel switching rate. This strategy puts comparable channel programming into similar categories such as news, movies, sports, music, and documentaries. This makes it easier for subscribers to identify the channels with similar aired programs among the vast number of available channels. The two-list scheme for managing two different lists of hot and cold channels is used to request channels and provides the subscribers with control over which channels to watch. This can save subscribers time spent searching for the required channel and eliminate the inadequacies of a recommendation system that may or may not suit the preferences of subscribers.

All channels in different categories are placed in the cold channel list. At the setup stage, subscribers can choose channels based on their preferences with the help of genre and channel descriptions to suit their channel preferences. The channels selected based on preference will be placed into a hot channel list, and such channels will remain in the hot channel list as long as subscribers keep viewing them; otherwise, they will be moved to the cold channel list. The advantage of the two-list scheme is that channel preferences can be updated at the will of a subscriber. The algorithm which recommends that programs of TV channels are scheduled on EPG is based on the most popular channels. Channel popularity is computed based on a per subscriber of channels in the hot channel list. The popularity of a channel $\left(P_{i}\right)$ in the hot channel list at a rate $\left(R_{i}\right)$ and for a given time $\left(T_{i}\right)$ is the product of the rate and time.

3.2. Personalized Electronic Program Guide. The personalized electronic program guide (EPG) bypasses typical monodirectional channel limits by providing subscribers with bidirectional customized channels of interest. These channels are selected in the hot channel list. The ability to customize EPG provides the subscribers with a level of assistance to find and watch programs of interest. The introduction of digital TV, IPTV, and an unprecedented volume of live TV channels available to subscribers has resulted in a new degree of information overload that results in channel zapping delay. The EPG that offers the customers of IPTV the capability to continuously update program data for the current and upcoming channel program events is viewed as a partial resolution to reduce channel switching time [21], which personalized EPG can resolve. It does so by eliminating the orthodox monodirectional channel limitations and offering the subscribers bidirectional personalized channels in the hot channel list that include only programs of interest to subscribers.

3.3. Explicit Semantic Analysis of Bag-of-Words. The explicit semantic analysis of bag-of-words (ESA-BOW) is a natural language processing (NPL) methodology for text categorization, semantic relatedness, and information retrieval that automatically derives a program description set of related concepts from large-scale knowledge resource repositories like Wikipedia [29-31]. The relationship between soap operas and television networks that broadcast them is, for instance, a well-studied topic in NLP for establishing relevance between the show and networks that broadcast it $[32,33]$. The ESA-BOW model was used to create a customized EPG based on keywords to improve rapid channel switching. It provides the advantage of helping to streamline the description of a keyword from a large number of related keywords. It can streamline keywords such as soap opera titles to a TV channel station for broadcasting. It accomplishes this by employing NLP techniques such as stop word removal and stemming to obtain the corresponding description for the keyword issued.

3.4. Really Simple Syndication. The really simple syndication (RSS) is a feed system that distributes Extensible Markup Language (XML) documents containing short descriptions of web updates to allow IPTV subscribers to subscribe to a feed of channels in the hot channel list they receive regularly for channel update [34-36]. The RSS feed can alert subscribers of an update in the IPTV system, while the model updates channel program descriptions with updated information from channels in a hot channel list. It attempts to 


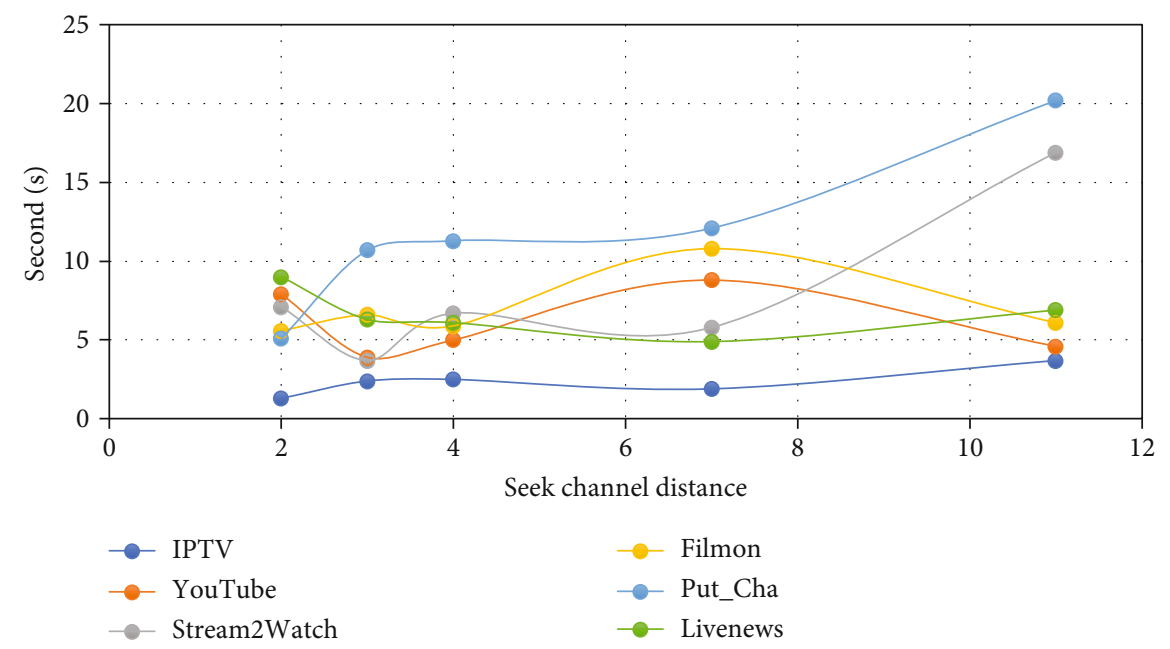

FIgURE 2: Time taken for channel switching.

reduce channel switching time and improve the quality of experience by granting subscribers autonomy based on their preferences. Any new IPTV system update will be publicized by adding it to the RSS feed. The RSS reader will poll the feed regularly and show the updates to the subscriber. However, there has been some debate in the literature as to whether RSS feeds contribute to IPTV zapping delay by consuming network capacity.

\section{Implementation}

This research has implemented the adaptive channel switching mechanism with customized EPG on a Raspberry Pi $3 \mathrm{~B}$ model using free IPTV channels. It is worth mentioning that these IPTV channels were exclusively utilized for instructional purposes and the sake of this study, with no plans for monetization. The final product was analyzed and compared against a variety of over the top (OTT) services and YouTube. Forty-five (45) channels from over 500 functioning channels available across various genres were chosen for the hot channel list, which can be found at https://pastebin.com/9rnRHnhx. This implementation was built on a converged network of the Durban University of Technology, and the experiment was carried out by one user for a month during peak and offpeak periods. The seek distance for channels was fixed between 2 and 11 [16, 17,37], and the experiment was carried out to determine the time it takes to switch from one channel to another. Figure 2 shows the comparison of the average time it takes to switch channels across five different platforms to the proposed approach over a month. The comparison to YouTube that uses a recommendation technique and other systems that use an approach based on the search engine has demonstrated that the proposed approach has the shortest channel switching time. Moreover, based on the results of this experiment, it is clear that the proposed IPTV allows the subscribers to determine their priorities based on personal desire rather than channel popularity or channel recommendation.
The experimental results of this study have generally shown that the proposed adaptive channel switching with a personalized program guide is suitable for addressing zapping delay. The experiment as shown in Figure 2 presents the seek channel distance from 2 channels to 11 channels. The livenews OTT has the highest time taken when the seek channel distance was set to 2 , followed by Youtube, stream2watch, Filmon, and put_cha, and IPTV recorded the lowest time taken while switching channels. The put_cha recorded the highest time taken when the seek channel distance was 3 , followed by Filmon, livenews, Youtube, and stream 2 watch and for the second time, IPTV recorded the lowest time taken while switching channels. Furthermore, when the seek channel distance was 4, put_cha maintained the most time taken, followed by Filmon, stream 2 watch, and Youtube, and IPTV maintained the lowest time taken for channel switching. Consequently, when the seek channel distance was 7 , put_cha maintained the most time taken, followed by Filmon, Youtube, stream 2 watch, and livenews, and IPTV recorded the lowest time taken while switching channels. It was noticed that time taken for Youtube channel switching was higher than usual. This delay can be attributed to the result that the desired channel was not part of the recommended channels, which is a major disadvantage of the recommendation system.

Finally, when the seek channel distance was 11, put_cha consistently maintained the most time taken, followed by stream2watch, livenews, Filmon, and YouTube, and IPTV recorded the lowest time taken while switching channels. However, it can be observed that IPTV recorded the highest time for channel switching at seek channel distance of 11 when compared to the seek channel distances at 2, 3, and 7. This is because of its capacity to prefetch channels into a hot channel list or cold channel list utilizing the two-list scheme. The excitement of this study is that the proposed IPTV outperformed the other OTT services tested in this study despite the limitation of increased time taken at a higher seek distance. Consequently, customers will be able to search for their favorite channels in a minimal amount of time. 


\section{Conclusion}

In this paper, we have used an adaptive channel switching strategy with a customized electronic program guide to producing quick channel navigation. The program was described using the customized EPG based on the natural language processing task of text classification utilizing the ESA-BOW algorithm. This strategy can help subscribers in selecting the preferred channels into the hot list of the proposed IPTV. Additionally, an RSS feed was used to assist subscribers in selecting a channel by notifying them of the available updates and changes. The proposed IPTV was ultimately implemented, tested, and deployed on Raspberry Pi 3B. The evaluation result of the proposed IPTV against five popular OTT service applications has indicated that it has outperformed others in terms of channel switching performance.

\section{Data Availability}

The dataset and implementation result data used to support the findings of this study have been deposited in the OSF repository (Adeliyi, T. T. (2020, January 21). Dataset retrieved from osf.io/pqs3h.

\section{Conflicts of Interest}

The authors declare that they have no conflicts of interest.

\section{References}

[1] D. A. Manzato and N. L. da Fonseca, "A survey of channel switching schemes for IPTV," IEEE Communications Magazine, vol. 51, no. 8, pp. 120-127, 2013.

[2] T. T. Adeliyi and O. O. Olugbara, "Fast channel navigation of internet protocol television using adaptive hybrid delivery method," Journal of Computer Networks and Communications, vol. 2018, 11 pages, 2018.

[3] C. Yang and Y. Liu, "On achieving short channel switching delay and playback lag in IP-based TV systems," IEEE Transactions on Multimedia, vol. 17, no. 7, pp. 1096-1106, 2015.

[4] J. Abreu, P. Almeida, B. Teles, and M. Reis, "Viewer behaviors and practices in the (new) television environment," in Proceedings of the 11th European conference on Interactive TV and video, pp. 5-12, Como, Italy, 2013.

[5] J. Zhang, Y. Li, M. Chen, and L. You, "An implicit feedback integrated LDA-based topic model for IPTV program recommendation.," in Proceedings of Communications and Information Technologies (ISCIT), 2016 16th International Symposium on, pp. 216-220, Qingdao, China, 2016.

[6] C. Yu, H. Ding, H. Cao, Y. Liu, and C. Yang, "Follow me: personalised IPTV Channel switching guide," in Proceedings of the 8th ACM on Multimedia Systems Conference, pp. 147157, Taipei, Taiwan, 2017.

[7] A. M. Elmisery, S. Rho, and D. Botvich, "Collaborative privacy framework for minimizing privacy risks in an IPTV social recommender service," Multimedia Tools and Applications, vol. 75, no. 22, pp. 14927-14957, 2016.

[8] H.-Y. Chang, S.-C. Huang, and C.-C. Lai, "A personalized IPTV channel-recommendation mechanism based on the
MapReduce framework," The Journal of Supercomputing, vol. 69, no. 1, pp. 225-247, 2014.

[9] M. Unger, A. Bar, B. Shapira, and L. Rokach, "Towards latent context-aware recommendation systems," Knowledge-Based Systems, vol. 104, pp. 165-178, 2016.

[10] A. A. Beyragh and A. G. Rahbar, "IFCS: an intelligent fast channel switching in IPTV over PON based on human behavior prediction," Multimedia Tools and Applications, vol. 72, no. 2, pp. 1049-1071, 2014.

[11] M. Manikandan, P. Saurigresan, and R. Ramkumar, "Grouped frequency interleaved ordering with pre-fetching for efficient channel navigation in internet protocol television," Multimedia Tools and Applications, vol. 75, no. 2, pp. 887-902, 2016.

[12] D.-J. Park and J.-D. Lee, "Implementation of Personalized EPG service based on Tru2Way," Journal of the Korea Industrial Information Systems Research, vol. 21, no. 1, pp. 51-59, 2016.

[13] N. M. Villegas, C. Sánchez, J. Díaz-Cely, and G. Tamura, "Characterizing context-aware recommender systems: a systematic literature review," Knowledge-Based Systems, vol. 140, pp. 173-200, 2018.

[14] S. Song, H. Moustafa, and H. Afifi, “Advanced IPTV services personalization through context-aware content recommendation," IEEE Transactions on Multimedia, vol. 14, no. 6, pp. 1528-1537, 2012.

[15] M. Krstic and M. Bjelica, "Context-aware personalized program guide based on neural network," IEEE Transactions on Consumer Electronics, vol. 58, no. 4, pp. 1301-1306, 2012.

[16] U. Oh, S. Lim, and H. Bahn, "Channel reordering and prefetching schemes for efficient IPTV channel navigation," IEEE Transactions on Consumer Electronics, vol. 56, no. 2, pp. 483487, 2010.

[17] E. Lee, J. Y. Ku, and H. Bahn, "An efficient hot channel identification scheme for IPTV channel navigation," IEEE Transactions on Consumer Electronics, vol. 60, no. 1, pp. 124-129, 2014.

[18] H. Bahn, "Channel reordering and prefetching techniques for efficient channel navigation in IPTVs," The Journal of the Institute of Internet, Broadcasting and Communication, vol. 16, no. 3, pp. 1-6, 2016.

[19] H.-S. Kim, I. Kim, K. Han, D. Kim, J.-S. Seo, and M. Kang, “An adaptive buffering method for practical HTTP live streaming on smart OTT STBs," KSII Transactions on Internet and Information Systems (TIIS), vol. 10, no. 3, pp. 1416-1428, 2016.

[20] H.-J. Kwon and K.-S. Hong, "Personalised electronic program guide for IPTV based on collaborative filtering with novel similarity method," in Proceedings of Consumer Electronics (ICCE), 2011 IEEE International Conference on, pp. 467-468, Las Vegas, NV, USA, 2011.

[21] F. Narducci, C. Musto, M. de Gemmis, P. Lops, and G. Semeraro, "TV-program retrieval and classification: a comparison of approaches based on machine learning," Information Systems Frontiers, vol. 20, no. 6, pp. 1157-1171, 2018.

[22] M. Cha, P. Rodriguez, J. Crowcroft, S. Moon, and X. Amatriain, "Watching television over an IP network," in Proceedings of the 8th ACM SIGCOMM conference on Internet measurement, pp. 71-84, Vouliagmeni, Greece, 2008.

[23] H. Uzunalioglu, "Channel change delay in IPTV systems," in Proceedings of Consumer Communications and Networking Conference, 2009. CCNC 2009, pp. 1-6, Las Vegas, NV, USA, 2009. 
[24] E. H. Khabbiza, R. El Alami, and H. Qjidaa, "A novel approach to reduce the unicast bandwidth of an IPTV system in a highspeed access network," International Journal of Digital Multimedia Broadcasting, vol. 2017, 2017.

[25] T. T. Adeliyi, R. E. Ogunsakin, M. O. Adebiyi, and O. O. Olugbara, "A meta-analysis of channel switching approaches for reducing zapping delay in internet protocol television," Indonesian Journal of Electrical Engineering and Computer Science, vol. 22, no. 3, p. 1476, 2021.

[26] N. Sailaja, A. Crabtree, and P. Stenton, "Challenges of using personal data to drive personalised electronic programme guides," in Proceedings of Proceedings of the 2017 CHI conference on human factors in computing systems, pp. 5226-5231, Denver, CO, USA, 2017.

[27] T. T. Adeliyi and O. O. Olugbara, "Reducing zapping delay in internet protocol television using a hybrid modular method," in 2019 Conference on Information Communications Technology and Society (ICTAS), pp. 1-4, Durban, South Africa, 2019.

[28] T. T. Adeliyi and O. O. Olugbara, "Designing two-list group program driven algorithm for channel navigation in internet protocol television," International Journal of Advanced Media and Communication, vol. 7, no. 4, p. 251, 2019.

[29] E. Gabrilovich and S. Markovitch, "Overcoming the Brittleness Bottleneck Using Wikipedia: Enhancing Text Categorization with Encyclopedic Knowledge," in Proceedings of AAAI, pp. 1301-1306, Boston, Massachusetts, USA, 2006.

[30] O. Egozi, E. Gabrilovich, and S. Markovitch, "Concept-Based Feature Generation and Selection for Information Retrieval," in Proceedings of $A A A I$, pp. 1132-1137, Chicago, Illinois, USA, 2008.

[31] J.-W. Son, A. Kim, and S.-B. Park, "A location-based news article recommendation with explicit localized semantic analysis," in Proceedings of Proceedings of the 36th international ACM SIGIR conference on Research and development in information retrieval, pp. 293-302, Dublin, Ireland, 2013.

[32] R. Baeza-Yates and B. Ribeiro-Neto, Modern Information Retrieval, ACM press New York, 1999.

[33] W. Wang, P. Chen, and B. Liu, "A self-adaptive explicit semantic analysis method for computing semantic relatedness using Wikipedia," in 2008 International Seminar on Future Information Technology and Management Engineering, pp. 3-6, Leicestershire, UK, 2008.

[34] J. Wusteman, "RSS: the latest feed," Library hi tech, vol. 22, no. 4, pp. 404-413, 2004.

[35] H. Liu, V. Ramasubramanian, and E. G. Sirer, "Client behavior and feed characteristics of RSS, a publish-subscribe system for web micronews," in Proceedings of Proceedings of the 5th ACM SIGCOMM conference on Internet Measurementpp. 1-3, Berkeley, California, USA, 2005.

[36] S. Kim and S. Lee, "Web technology and standardization for web 2.0 based IPTV service," in 2008 10th International Conference on Advanced Communication Technology, pp. 17511754, Gangwon, Korea (South), 2008.

[37] Y. W. Chen and T. T. Chiu, "Minimizing zapping time in IPTV based on user's interest," International Journal of Computer and Communication Engineering, vol. 1, no. 2, pp. 77$80,2012$. 